\title{
Modelling of atorvastatin pharmacokinetics and the identification of the effect of a BCRP polymorphism in the Japanese population
}

DOI:

10.1097/FPC. 0000000000000252

\section{Document Version \\ Accepted author manuscript}

Link to publication record in Manchester Research Explorer

Citation for published version (APA):

Tsamandouras, N., Guo, Y., Wendling, T., Hall, S., Galetin, A., \& Aarons, L. (2017). Modelling of atorvastatin pharmacokinetics and the identification of the effect of a BCRP polymorphism in the Japanese population. Pharmacogenetics and Genomics, 27(1), 27-38. https://doi.org/10.1097/FPC.0000000000000252

\section{Published in:}

Pharmacogenetics and Genomics

\section{Citing this paper}

Please note that where the full-text provided on Manchester Research Explorer is the Author Accepted Manuscript or Proof version this may differ from the final Published version. If citing, it is advised that you check and use the publisher's definitive version.

\section{General rights}

Copyright and moral rights for the publications made accessible in the Research Explorer are retained by the authors and/or other copyright owners and it is a condition of accessing publications that users recognise and abide by the legal requirements associated with these rights.

\section{Takedown policy}

If you believe that this document breaches copyright please refer to the University of Manchester's Takedown Procedures [http://man.ac.uk/04Y6Bo] or contact uml.scholarlycommunications@manchester.ac.uk providing relevant details, so we can investigate your claim.

\section{OPEN ACCESS}




\section{Modelling of atorvastatin pharmacokinetics and the identification of the effect of a BCRP polymorphism in the Japanese population}

Nikolaos Tsamandouras ${ }^{1}$, Yingying Guo ${ }^{2}$, Thierry Wendling ${ }^{1}$, Stephen Hall ${ }^{2}$, Aleksandra Galetin $^{1}$, Leon Aarons ${ }^{1}$

1. Centre for Applied Pharmacokinetic Research, Manchester Pharmacy School, University of Manchester, Manchester, UK.

2. Eli Lilly and Company, Indianapolis, Indiana, USA.

Running head: Atorvastatin PK in the Japanese population

Corresponding Author / Request for Reprints (current affiliation): Nikolaos Tsamandouras, Department of Biological Engineering, Massachusetts Institute of Technology, 77 Massachusetts Avenue, Building 16, Room 16-451, Cambridge, Massachusetts, USA, Tel: +1 617324 6065, Fax: +1 617253 2400, Email address: ntsaman@mit.edu

Conflict of interest and Source of Funding: During this work NT was an employee of the University of Manchester and currently employed by the Massachusetts Institute of Technology. YG and SH are employees of Eli Lilly and Company. AG and LA are employees of the University of Manchester. During this work TW was a PhD student at the University of Manchester. No other relationships or activities were declared from the authors that could appear to have influenced the submitted work. 


\begin{abstract}
$\operatorname{Aim}(\mathbf{s}):$ Ethnicity has a modulating role in atorvastatin pharmacokinetics, with Asian subjects reported to have higher exposure compared to Caucasians. Therefore, it is difficult to safely extrapolate atorvastatin pharmacokinetic data and models across ethnic groups. This work aims to develop a population pharmacokinetic model for atorvastatin and its pharmacologically active metabolites specifically for the Japanese population. Subsequently it is aimed to identify genetic polymorphisms affecting atorvastatin pharmacokinetics in this population.
\end{abstract}

Methods: Atorvastatin acid and o-OH-atorvastatin acid plasma concentrations, clinical/ demographic characteristics and genotypes for 18 genetic polymorphisms $(3,3,1,1,7,2$ and 1 in the $A B C B 1, A B C G 2, C Y P 3 A 4, C Y P 3 A 5, S L C O 1 B 1, S L C O 2 B 1$ and PPARA genes respectively) were collected from 27 Japanese individuals (taking 10mg atorvastatin once daily) and analysed using a population pharmacokinetics modelling approach.

Results: The developed population pharmacokinetic model (one-compartment for atorvastatin acid linked through metabolite formation to an additional compartment describing the disposition of o-OH-atorvastatin acid) accurately described the observed data and the associated population variability. Our analysis suggested that subjects carrying one variant allele for the rs2622604 polymorphism (ABCG2) exhibit a 55\% (95\%CI: $16 \%$-131\%) increase in atorvastatin oral bioavailability relatively to the value in individuals without the variant allele.

Conclusion: The current work reports the identification in the Japanese population of a BCRP polymorphism, not previously associated with the pharmacokinetics of any statin, that substantially increases atorvastatin acid and o-OH-atorvastatin acid exposure. The developed model may be of clinical importance in order to guide dosing recommendations tailored specifically for the Japanese. 
Keywords: atorvastatin, pharmacokinetics, pharmacogenetics, Japanese, ABCG2, BCRP

\section{Introduction}

Atorvastatin belongs to the class of HMG-CoA reductase inhibitors (statins) and is currently one of the most commonly used medications to treat lipid disorders and prevent cardiovascular events in high-risk individuals [1-3]. Similarly to other statins, atorvastatin has been associated with the development of skeletal muscle toxicity (myopathy). The severity of the latter can range from myalgia (muscle pain with no evidence of muscle degradation) to the rare but life-threatening rhabdomyolysis (severe muscle damage potentially accompanied with acute renal failure) [4-8]. It is widely acknowledged that statin-induced myopathy is a concentration-dependent adverse event and that factors such as increased dose and coadministration of compounds altering statin pharmacokinetics substantially increase myopathy risk $[9,10]$. Therefore, in-depth understanding of atorvastatin pharmacokinetics and the potential effect of clinical, demographic and genetic factors are of significant importance.

Atorvastatin is administered in its active acid form, atorvastatin acid (ATA), and it is predominantly metabolised in the liver and gut wall by CYP3A4 $[1,11]$. It has a low $(14 \%)$ oral bioavailability (F) due to the extensive first-pass metabolism and its renal elimination is negligible [11]. ATA has two major CYP3A metabolites, ortho-hydroxy-atorvastatin acid (oOH-ATA) and para-hydroxy-atorvastatin acid (p-OH-ATA), which are also active. Specifically, it has been reported that these active metabolites account for approximately $70 \%$ of the circulating HMG-CoA reductase inhibitory activity $[1,11]$. Both ATA and its active CYP3A metabolites can undergo lactonisation (partly via the formation of acyl-glucuronide intermediates) and thus in vivo they exist in equilibrium with their respective inactive lactone forms [11-13]. 
Polymorphisms in disposition-related genes have been reported to affect the pharmacokinetics of atorvastatin. Specifically, Pasanen et al [14] identified that a wellstudied polymorphism (c.521T $>\mathrm{C}$ or rs4149056) in the gene coding for the OATP1B1 hepatic uptake transporter $(S L C O 1 B 1)$ increases ATA exposure by $144 \%$ in individuals with the homozygous variant genotype (CC) compared to homozygous wild-type individuals (TT). This polymorphic effect is attributed to the decreased hepatic uptake and subsequently metabolism of ATA that leads to higher systemic concentrations. In a different study [15] a polymorphism (c.421C $>\mathrm{A}$ or rs2231142) in the gene coding for the BCRP efflux transporter ( $A B C G 2)$ was reported to be associated with $72 \%$ increased ATA exposure in homozygous variant individuals (AA) compared to wild-type individuals (CC). The increased ATA exposure in the polymorphic subjects has been suggested to occur due to decreased efflux on the intestinal wall and subsequently higher bioavailability after oral administration.

Ethnicity has been also identified to have a modulating role in atorvastatin pharmacokinetics, as Asian subjects have been reported to have significantly higher exposure to ATA compared to Caucasians [16]. This specific ethnic effect has been also observed with regard to other statin acid forms such as rosuvastatin [16, 17] and simvastatin acid [16, 18]. Characteristically, in the U.S. the recommended rosuvastatin initial dose in Asians is half of the dose recommended in Caucasians [19]. Available data suggest that this ethnic variability is due to intrinsic rather than extrinsic such as environmental factors, as it exists even between individuals in the same environment $[17,20]$. However, this ethnic variability cannot be fully attributed to differences in the population allele frequencies of the up-to-date known clinically important polymorphisms (e.g. SLCO1B1 c.521T >C, ABCG2 c.421C >A) affecting the PK of statins $[16,18,19]$. It has been proposed that this ethnic effect arises due to lower OATP1B1 hepatic expression in Asians compared to Caucasians irrespectively of the SLCO1B1 genotype [19]; however recent proteomic findings are contradicting this theory 
[21]. Understanding the origin of this observed ethnic variability in statin exposure is an area of ongoing research that has received substantial effort lately. However, with the current knowledge it is very difficult to safely extrapolate atorvastatin pharmacokinetic data and models across ethnic groups (specifically from Caucasians to Asians).

Therefore, the aim of this work is to develop a population pharmacokinetic model for ATA and its pharmacologically active metabolites specifically for the Japanese population, which is to our knowledge currently lacking. Subsequently, it is aimed to identify specific single nucleotide polymorphisms (SNPs) that affect the pharmacokinetics of ATA and its active metabolites in this population.

\section{Methods}

\section{Description of the available data for model development}

The atorvastatin pharmacokinetic data presented in the current work emerged from a clinical trial sponsored by Eli Lilly that aimed to show efficacy of LY2484595 either as monotherapy or in combination with atorvastatin in patients with dyslipidemia. The data reported in the current work are from an arm of this trial in which only atorvastatin was given to 27 Japanese patients (10 mg orally once daily for 12 weeks). The study protocol was reviewed and approved by ethical review boards (Supplemental Digital Content 1, section 1). The study was conducted in accordance with all applicable regulatory and Good Clinical Practice guidelines and followed the ethical principles originating in the Declaration of Helsinki. All subjects signed an informed consent document prior to participation in the study. Extensive details regarding the clinical study, such as all the primary and secondary outcome measures, study arms and inclusion/exclusion criteria can be found by accessing ClinicalTrials.gov (study identifier NCT01375075). The studied individuals were not receiving any concomitant 
medications that could affect the pharmacokinetics of ATA or its major active metabolites (oOH-ATA and p-OH-ATA). Among all patients only one had previous lipid modifying therapy within the past year prior to the study and the medication was Crestor tablets $2.5 \mathrm{mg}$.

Blood samples were collected on 4 different visits to the clinical research unit and specifically on weeks $2,4,8$ and 12 since the beginning of the study. To record the time of atorvastatin intake on the day of the pharmacokinetic measurements, subjects were instructed not to take the daily dose at home but at the investigator site. The sampling was sparse in that only 2 samples were taken per visit (1 pre-dose and 1 post-dose). However, the design of the study was such that the post-dose sample was taken at different time at every visit, in order to obtain more information regarding the entire pharmacokinetic profile of the analysed compounds. Illustratively, for a study participant (subject ID: 401), the post-dose sample on week 2, 4, 8 and 12 was taken at 1, 3, 6 and 8 hours after dose respectively. The pre-dose blood samples were drawn to determine trough concentrations of the analysed compounds (the majority of the pre-dose samples were drawn 22-29 hours since the last reported dose). Compliance with the required dosing regimen was assessed by direct questioning and by counting returned study drug. A patient was considered significantly non-compliant if he/she missed more than 7 consecutive days of study medication, or more than 16 cumulative days of study medication during the entire study. No patients were significantly non-compliant.

In total 216 venous blood samples were collected. These samples were drawn into heparincoated tubes, which were then subsequently used to isolate plasma samples. Plasma samples were immediately frozen and kept frozen at approximately $-20^{\circ} \mathrm{C}$ or lower until the time of the assay. Samples were analysed to determine ATA, o-OH-ATA and p-OH-ATA concentrations using a validated LC/MS/MS method (Supplemental Digital Content 1, 
section 2). All 8 samples from one patient had to be excluded from the pharmacokinetic analysis due to quantification issues.

All the demographic and clinical characteristics of the studied participants are summarised in Table 1. For the purpose of this work, all studied participants were retrospectively genotyped for 18 genetic polymorphisms located in 7 different genes. More specifically 3, 3, 1, 1, 7, 2 and 1 polymorphisms were genotyped in the $A B C B 1, A B C G 2, C Y P 3 A 4, C Y P 3 A 5, S L C O 1 B 1$, SLCO2B1 and PPARA genes respectively, as previous literature suggests that all these genes might be implicated in atorvastatin disposition $[14,15,22-26]$. The distribution of these variants in the studied population is reported in Table 2. Details of the genotyping and quality control (QC) methods are reported in Supplemental Digital Content 1 (section 3).

\section{Population pharmacokinetic analysis}

Population pharmacokinetic modelling was performed using nonlinear mixed effects modelling software (NONMEM ${ }^{\circledR}$ 7.3, ICON Development Solutions, Ellicott City, Maryland, USA) and the first order conditional estimation method with interaction (FOCE-I). Additional investigations of the NONMEM output, statistical and graphical analysis was performed in Matlab R2014a (The MathWorks, Inc., Natick, Massachusetts, USA).

\section{Structural and statistical model}

The ATA, o-OH-ATA and p-OH-ATA plasma concentrations available for pharmacokinetic analysis are presented in Figure 1. In total, 41/208 (19.7\%), 12/208 (5.8\%) and 130/208 (62.5\%) plasma concentration measurements were below the limit of quantification (BQL) for ATA, o-OH-ATA and p-OH-ATA respectively. Due to the substantial proportion of BQL data for p-OH-ATA, population modelling of this particular metabolite could not be informed from the available data in this study. A literature search was performed to identify any 
previous population pharmacokinetic studies in which p-OH-ATA was measured in order to obtain priors that can support the modelling of this metabolite. In the only (to our knowledge) population pharmacokinetic study that $\mathrm{p}-\mathrm{OH}-\mathrm{ATA}$ was measured, more than $80 \%$ of the measurements were BQL and thus, similarly to here, this metabolite was not modelled [27]. Therefore, population pharmacokinetic modelling was finally pursued only with regard to ATA and its measurable active metabolite o-OH-ATA. As the proportion of BQL data is relatively low for ATA and o-OH-ATA, any such data were treated as missing and were excluded from the analysis [28]. This approach was further validated with the use of a visual predictive check (VPC) variation in which the agreement between the fraction of observed and model-predicted BQL concentrations is also examined [29].

The available ATA and o-OH-ATA plasma concentrations were expressed in their molar equivalents (molecular weight of ATA and o-OH-ATA is 558.64 and 574.64 respectively) and were log-transformed for simultaneous parent-metabolite modelling. Several structural parent-metabolite pharmacokinetic models were considered including different combinations of one-, two- and three-compartment disposition models for ATA and o-OH-ATA. The elimination of both ATA and o-OH-ATA was assumed to occur from the central compartments. Different simple absorption models were considered (e.g. first-order absorption), as the evaluation of more complex models was constrained by the limited amount of data during the absorption phase of ATA (Figure 1). The joint parent-metabolite models are structurally not identifiable, since ATA and o-OH-ATA plasma data after administration of the parent drug (ATA) are only available. Assuming complete conversion of ATA to o-OH-ATA or equal volume of distribution for the two compounds can resolve identifiability issue but it would be physiologically invalid and would introduce bias in our results. Therefore parameter estimation and subsequent covariate analysis was performed in the domain of the identifiable "apparent" parameters (Results). 
Inter-individual variability (IIV) random effect terms were assigned on model parameters using an exponential relationship. Correlation between the IIV random effect components was also explored by the incorporation of covariance elements (OMEGA BLOCK option in NONMEM). The incorporation of inter-occasion variability (IOV) [30] was also evaluated in model parameters and was retained as long the objective function and model diagnostics were improved. Illustratively, IIV and IOV was incorporated on a model parameter $\theta$ according to Eq.1.

$$
\theta_{i j}=\tilde{\theta} \cdot e^{\eta_{i}+\kappa_{j}}
$$

where $\theta_{i j}$ is the parameter estimate referring to the $\mathrm{i}^{\text {th }}$ individual and the $\mathrm{j}^{\text {th }}$ occasion (a different visit to the clinical research unit is considered as occasion), $\tilde{\theta}$ is the typical population parameter value, $\eta_{i}$ is the random effect referring to differences between individuals and $\kappa_{j}$ is the random effect referring to between occasion differences within an individual (both $\eta$ and $\kappa$ are assumed to be normally distributed with a mean of zero). A common variance was assumed between occasions using the SAME option in OMEGA BLOCK. The residual unexplained variability was modelled for both ATA and o-OH-ATA with an additive error model on the log-transformed data.

\section{Covariate model building}

The following population covariates were available and investigated during the covariate model building procedure: gender, age, height, weight, body mass index (BMI), alcohol/ caffeine/ tobacco products consumption, ALT (alanine transaminase) and AST (aspartate transaminase) liver function tests and genotypes for the 18 SNPs reported in Table 2. Techniques for the incorporation of multiple SNPs in nonlinear mixed effects models have been recently proposed and compared [31] including both stepwise forward inclusion backward elimination procedures [32] and penalised regressions [33]. In this study, the 
number of tested SNPs is relatively small and a computationally intensive stepwise procedure (extensively described in a previous work [18]) was employed for covariate model building. Briefly, this procedure involves screening of empirical Bayes estimates (given low $\eta$ shrinkage) and a stepwise forward inclusion - backward elimination algorithm that also examines the degree of linkage disequilibrium (LD) between correlated SNPs upon inclusion in each step. Linkage disequilibrium between closely linked SNPs (e.g. the 7 SNPs analysed in the SLCOIBI gene) was assessed in the studied population (with the R-squared metric) and then visually inspected with snp.plotter [34]. The statistical significance threshold was set to 0.05 for the forward inclusion and 0.005 for the backward elimination steps (assessed by a likelihood ratio test (LRT) between the models with or without the covariate). In addition, as the sample size in the current work is relatively small, a permutation (randomisation) testing procedure [35] was employed in order to take into account the possibility of type-I error inflation and correct the LRT significance levels during the backward elimination process (Supplemental Digital Content 1, section 4).

A bootstrapping procedure $(n=1,000)$ was performed with PsN 3.7.6 (Perl-speaksNONMEM) [36] in order to evaluate the robustness of parameter estimates and obtain nonparametric $95 \%$ confidence intervals around these values.

\section{Results}

The model that best described the plasma concentrations of both ATA and o-OH-ATA is presented in Figure 2. It consists of a first-order absorption process and a 1-compartment disposition model for ATA, which is linked through metabolite formation to an additional compartment that describes the disposition of o-OH-ATA. Alternative parent-metabolite model structures (different combinations of one or two compartment disposition models for ATA and o-OH-ATA) either resulted in model misspecification or did not offer any 
significant improvement in the objective function and model diagnostics compared to the selected model. The set of identifiable parameters in the presented pharmacokinetic model are: $\mathrm{k}_{\mathrm{a}}$ (first-order absorption rate constant), $\mathrm{CL}_{\mathrm{ATA}} / \mathrm{F}$ (apparent ATA total elimination clearance), $\mathrm{V}_{\mathrm{ATA}} / \mathrm{F}$ (apparent ATA volume of distribution), $\mathrm{F} \cdot \mathrm{f}_{\mathrm{m}} / \mathrm{V}_{\mathrm{o}-\mathrm{OH}-\mathrm{ATA}}$ (apparent fraction metabolised to o-OH-ATA), $\mathrm{CL}_{\mathrm{o}-\mathrm{OH}-\mathrm{ATA}} / \mathrm{V}_{\mathrm{o}-\mathrm{OH}-\mathrm{ATA}}$ (apparent o-OH-ATA total elimination clearance). The parameter estimates of the final population pharmacokinetic model together their 95\% confidence intervals are reported in Table 3. The NONMEM estimates were in close agreement with the medians obtained from the bootstrap samples for all model parameters. The final population model included IIV random effect terms in all the identifiable model parameters (listed above) and covariance elements between the $\mathrm{CL}_{\mathrm{ATA}} / \mathrm{F}$ and $\mathrm{V}_{\mathrm{ATA}} / \mathrm{F}$ and between the $\mathrm{F} \cdot \mathrm{f}_{\mathrm{m}} / \mathrm{V}_{\mathrm{o}-\mathrm{OH}-\mathrm{ATA}}$ and $\mathrm{CL}_{\mathrm{o}-\mathrm{OH}-\mathrm{ATA}} / \mathrm{V}_{\mathrm{o}-\mathrm{OH}-\mathrm{ATA}}$ IIV terms (Table 3 ). Inclusion of IOV on bioavailability substantially improved the objective function and model diagnostics and thus was retained in the final model.

Typical goodness of fit plots for the final population pharmacokinetic model are presented in Figures 3 and 4 referring to ATA and o-OH-ATA plasma concentrations respectively. A visual predictive check $(n=1,000)$ of the final population model is presented in Figure 5. It is evident that the developed joint parent-metabolite model adequately reflects the observed data and their variability for both ATA and o-OH-ATA.

The effect of the rs2622604 genotype ( $A B C G 2$ gene) was included in the final model. Specifically, our analysis suggested that individuals carrying one variant allele for this SNP (AG genotype) have 55\% higher bioavailability (95\% CI: $16 \%-131 \%)$ relatively to the value in homozygous wild-type individuals (GG). It should be noted that due to the lack of IV data, absolute bioavailability values cannot be determined across the different genotypes and thus covariate effects on bioavailability are manifested/identified in the domain of apparent parameters (e.g. $\mathrm{CL}_{\mathrm{ATA}} / \mathrm{F}, \mathrm{V}_{\mathrm{ATA}} / \mathrm{F}$ ). The empirical Bayes estimates of all the apparent 
parameters that incorporate a bioavailability term $\left(\theta_{2}-\theta_{4}\right.$, Table 3$)$ substantially differed between individuals with the variant (AG) and the wild type genotype (GG). Based on physiological knowledge of the SNP effect, (decreased BCRP expression in the intestine, see Discussion) it was interpreted that it is the bioavailability that is affected by this polymorphism rather than the other PK parameters themselves (e.g. CL $L_{\mathrm{ATA}}, \mathrm{V}_{\mathrm{ATA}}$ ). Subsequently, a unique covariate effect $\left(\theta_{6}\right.$, Table 3$)$ was incorporated into the bioavailability terms of the apparent model parameters, corresponding to the fractional change in oral bioavailability in individuals carrying one variant allele relatively to the bioavailability in homozygous wild-type individuals. Inclusion of this covariate effect in the model significantly improved the description of the observed data. The $55 \%$ increased bioavailability in the variant genotype individuals yields an equivalent increase in the modelsimulated plasma exposures of ATA and o-OH-ATA. A visual predictive check stratified by the rs2622604 genotype is presented in Figure 6. It is evident that this BCRP polymorphism increases (on average) the plasma concentrations of both ATA and o-OH-ATA. The developed model that incorporates this SNP effect on atorvastatin bioavailability was able to adequately describe the observed data and their variability not only in total (Figure 5) but also within each genotype population (Figure 6).

Removal of this SNP effect from the model during the backwards elimination step of the model building procedure increased the objective function value by 17.46 units illustrating the importance of this covariate. The permutations-generated empirical distribution of the LRT statistic (change in objective function value) was used to calculate the corresponding significance level instead of the $\chi^{2}$ distribution in order to guard against Type-I error inflation. It was evident from the results of the permutation procedure that substantial Type-I error inflation can occur in such a small sample size if the LRT statistic is assumed to follow the $\chi^{2}$ distribution (Figure 7). Specifically, the $\chi^{2}$ probability density is shifted to the left 
compared to the empirical distribution of the test statistic under the null hypothesis and thus produces consistently lower p-values. Characteristically, the 17.46 units change in the objective function value, when the rs2622604 SNP is removed from the model, corresponds to a p-value of $2.9 \cdot 10^{-5}$ if the $\chi^{2}$ distribution is assumed for the LRT statistic. In contrast, using the empirical distribution of the test statistic the same change in objective function yields the permutation-corrected p-value $\left(p_{\text {perm }}\right)$ of 0.0045 , which was the one used to assess the SNP significance. As this value was below the pre-defined statistical threshold (0.005) the effect of the rs2622604 SNP was retained in the model. This SNP was not identified to have a significant effect on the absorption rate constant $\left(\mathrm{k}_{\mathrm{a}}\right)$ of the drug. The other 2 SNPs tested in the $A B C G 2$ gene (rs2231142 and rs4148162) were also not identified to have a significant effect.

The linkage disequilibrium pattern between the 7 SNPs tested in the SLCO1B1 gene in the studied Japanese population is illustrated in Figure 8. It was evident that in this population the rs4149056 SNP is inherited almost independently of the other 6 SLCO1B1 tested SNPs, whilst the latter are in strong correlation with each other. However, neither rs4149056 nor the other 6 SNPs were found to significantly affect ATA/o-OH-ATA model parameters in the population studied. Similarly, none of the SNPs tested in the $A B C B 1, C Y P 3 A 4, C Y P 3 A 5$, PPARA and SLCO2B1 genes or any of the available non-genetic covariates (e.g. age, gender) had a substantial effect on model parameters. Summarizing the covariate model building procedure, the $A B C G 2$ rs 2622604 SNP was the first covariate to be included in the model (as it produced the strongest signal through the initial covariate screening) and afterwards no other covariates achieved the required statistical significance levels for model inclusion. 


\section{Discussion}

Recent clinical study [16] has shown that Japanese have significantly different atorvastatin PK parameters (AUC, Cmax) compared to Caucasians. Consequently, previous population pharmacokinetic models for atorvastatin [37, 38] (developed mainly with data from Caucasians) cannot be safely extrapolated in the Japanese population. To our knowledge, the current work is the first to report a population pharmacokinetic model for atorvastatin that specifically focuses on the Japanese population. The fact that the developed model jointly describes the kinetics not only of ATA but also of its active metabolite, o-OH-ATA, is of particular importance in case that propagation to the pharmacodynamic effects is also desirable.

A further objective of this work was to identify genetic covariates that have a substantial impact on atorvastatin pharmacokinetics in the Japanese population. In contrast to a noncompartmental data analysis approach, the use of nonlinear mixed effects modelling allows the investigation of genetic effects even in the case where sparse data are available (as in the current study). Additional advantages also arise in a model-based approach such as increased statistical power to detect a genetic effect and improved interpretation of its underlying mechanism [31]. However, it should be clearly noted that in order to enjoy all the benefits from such an approach, studies that are adequately designed in terms of sample size and sampling times are needed.

Our results suggest that a polymorphism (rs2622604) in the $A B C G 2$ gene substantially increases atorvastatin oral bioavailability and subsequently plasma exposure to both ATA and o-OH-ATA. The rs2622604 is an intronic SNP and in vitro it has been previously identified to decrease BCRP expression in the intestine [39]. This supports the findings of the current study, as the lower intestinal BCRP expression in individuals carrying the variant rs2622604 
genotype will lead to decreased efflux of the drug back to the intestinal lumen and thus higher bioavailability. This SNP has never been associated before with the pharmacokinetics of atorvastatin or any other statin in vivo. However, it has been repeatedly linked with the pharmacokinetics/pharmacodynamics of compounds used in cancer therapeutics. Specifically, in a previous clinical study the rs2622604 SNP was associated with irinotecaninduced myelosuppression, proposed to occur consequently to higher levels of the drug in the systemic circulation [40]. In addition, this SNP has been associated with increased plasma exposure to erlotinib [41] and higher risk for the development of gefitinib [42] and sunitinib [43] induced toxicity/ adverse events. The fact that an association between the rs 2622604 genotype and statin kinetics was identified in this work is of particular importance, as up to now most of the BCRP-related pharmacogenetic research in this class of drugs focuses on the effects of the c.421C $>$ A polymorphism.

Neither the c.421C $>\mathrm{A}(A B C G 2)$ nor the c.521T $>\mathrm{C}(S L C O 1 B 1)$ polymorphisms were identified to affect atorvastatin pharmacokinetics in the current work. The effects of these two polymorphisms on atorvastatin pharmacokinetics have been robustly identified in Caucasian populations $[14,15]$ and were also recently illustrated in Japanese subjects [16]. It can be hypothesised that the lack of effect in the current work may be due to the small sample size and the complete absence of homozygous variant individuals with respect to these SNPs (Table 2). Similarly, the effects of two other SNPs that have been of particular clinical interest recently, namely rs35599367 (CYP3A4) and rs4253728 (PPARA) [18, 23, 26], were not possible to be investigated in the current work as all studied individuals carried the homozygous wild-type genotype (Table 2).

Apart from the well-characterised c.521T $>$ C polymorphism, a group of 6 additional SNPs were also genotyped in the SLCO1B1 gene (rs11045879, rs2291075, rs4149048, rs4149050, rs4149081 and rs4363657). Specifically, three of these SNPs have been previously associated 
either with altered methotrexate clearance (rs4149081, rs11045879) $[44,45]$ or simvastatininduced myopathy (rs4363657) [46] and they occur in a much higher frequency in Asian populations compared to Caucasians [47]. Therefore, the reported ethnic variability in statin kinetics could be potentially attributed to the effects of these SNPs. However, these three SNPs are in strong LD with the c.521T $>$ C SNP (rs4149056) in Caucasian populations, thus it is difficult to identify in vivo if they carry any additional effects independent of the c.521T $>\mathrm{C}$ SNP. In contrast, a completely different LD pattern occurs in the Japanese (Figure 8) where all the 6 SLCO1B1 SNPs reported above are in high LD with each other but are inherited almost independently of the c.521T $>$ C SNP. Therefore, the independent causal effects of this group of SLCO1B1 SNPs on atorvastatin pharmacokinetics could be investigated in this work. Nevertheless, no evidence of association was observed between the genotypes in this group of 6 SNPs and atorvastatin pharmacokinetics. Similarly, in our previous study regarding simvastatin acid pharmacokinetics [18], a functional effect (independent of the c.521T $>$ C polymorphism) could not be attributed to these SNPs. Taken together the results of this previous study and the current work suggest that it is unlikely that these SNPs exert a strong functional effect on the kinetics of OATP1B1-substrates that can possibly explain the reported ethnic variability.

It has been recently suggested that this ethnic variability is more likely to be due to a BCRPrelated effect during the absorption process, rather than due to reduced OATP1B1-mediated hepatic uptake in Asians [16, 21]. It should be clearly noted that the BCRP polymorphism (rs2622604) identified in this work to increase exposure to both atorvastatin and its active metabolite is not expected to explain the observed ethnic variability as it does not have a higher variant allele frequency in Japanese compared to Caucasians [47]. However, this finding clearly highlights the importance of BCRP transporter on atorvastatin pharmacokinetics in the Japanese population. 
The sample size of the current study is one of the main limitations of this work. However, atorvastatin PK data in Japanese dyslipidemic patients in conjunction with genotypes for several SNPs in different genes implicated in atorvastatin disposition are not readily available in the literature. Therefore, this work aims to expand the current literature knowledge and generate data and hypotheses, which can be valuable for the design of future studies in this population. Besides that, only genetic variants which can be supported by previous in vitro / in vivo findings were tested in this work and it was our intention to explore SNP effects of large magnitude which may suggest the need for dose adjustment. As the LRT that was used here to detect significant genotype effects is an asymptotic test, substantial type-I error inflation may arise with small sample size and/or unevenly distributed genotypes [35, 48, 49]. Therefore, a permutation procedure was performed in order to correct significance levels for Type-I error inflation. The fact that the rs2622604 (ABCG2) SNP remains significant even after permutation correction provides additional confidence that it represents a genuine genotype effect and not a spurious association. Future studies are needed that replicate the polymorphic effect identified in this work and also assess its relevance in other ethnic populations.

The model reported here was adequate for the purpose of investigation (prediction of ATA/oOH-ATA plasma levels in Japanese individuals with different genotypes), however its empirical structure and data-driven nature can be considered as a limitation. Indicatively, the model does not incorporate any pre-systemic formation of o-OH-ATA and more importantly does not allow concentration predictions directly at the drug's site of action (liver) or site of toxicity (muscle). The sparsity of the available pharmacokinetic data restricted any attempts to evaluate more complex mechanistic model structures. However, if more data become available in the future they should be integrated with prior information deriving from the physiological understanding of the studied system and in vitro experiments, in order to 
develop a mechanistically more sound atorvastatin model, as exemplified in recent studies [50-52].

In conclusion, we presented here the first population pharmacokinetic model for atorvastatin that specifically focuses on the Japanese population. The current work reports the identification in this population of a polymorphism in the $A B C G 2$ gene that substantially increases the exposure to both ATA and its active metabolite o-OH-ATA and which should be subject of future studies. Taking into account the substantial ethnic variability in atorvastatin kinetics and the fact that elevated systemic drug concentrations may increase myopathy risk, it becomes apparent that this work can be of clinical importance in order to design future pharmacogenetic studies and guide dosing recommendations tailored specifically for the Japanese population.

\section{Acknowledgements}

The authors would like to acknowledge the fruitful comments and discussions made by the members of the Centre for Applied Pharmacokinetic Research at the University of Manchester.

\section{References}

1. Lea A, McTavish D. Atorvastatin. Drugs. 1997; 53: 828-847.

2. Bakker-Arkema RG, Best J, Fayyad R, Heinonen TM, Marais AD, Nawrocki JW, et al. A brief review paper of the efficacy and safety of atorvastatin in early clinical trials. Atherosclerosis. 1997; 131: 17-23.

3. Sillesen H, Amarenco P, Hennerici MG, Callahan A, Goldstein LB, Zivin J, et al. Atorvastatin reduces the risk of cardiovascular events in patients with carotid 
atherosclerosis: A secondary analysis of the stroke prevention by aggressive reduction in cholesterol levels (SPARCL) trial. Stroke. 2008; 39: 3297-3302.

4. Ramsey LB, Johnson SG, Caudle KE, Haidar CE, Voora D, Wilke RA, et al. The Clinical Pharmacogenetics Implementation Consortium guideline for SLCO1B1 and simvastatin-induced myopathy: 2014 update. Clin Pharmacol Ther. 2014; 96: 423428.

5. Thompson PD, Clarkson P, Karas RH. Statin-associated myopathy. JAMA. 2003; 289: $1681-1690$.

6. Graham DJ, Staffa JA, Shatin D, Andrade SE, Schech SD, La Grenade L, et al. Incidence of hospitalized rhabdomyolysis in patients treated with lipid-lowering drugs. JAMA. 2004; 292: 2585-2590.

7. Thompson PD, Clarkson PM, Rosenson RS. An assessment of statin safety by muscle experts. Am J Cardiol. 2006; 97: S69-S76.

8. McKenney JM, Davidson MH, Jacobson TA, Guyton JR. Final conclusions and recommendations of the national lipid association statin safety assessment task force. Am J Cardiol. 2006; 97: S89-S94.

9. Omar MA, Wilson JP, Cox TS. Rhabdomyolysis and HMG-CoA reductase inhibitors. Ann Pharmacother. 2001; 35: 1096-1107.

10. Wilke RA, Ramsey LB, Johnson SG, Maxwell WD, McLeod HL, Voora D, et al. The clinical pharmacogenomics implementation consortium: CPIC guideline for SLCO1B1 and simvastatin-induced myopathy. Clin Pharmacol Ther. 2012; 92: 112117.

11. Lennernas H. Clinical pharmacokinetics of atorvastatin. Clin Pharmacokinet. 2003; 42: 1141-1160. 
12. Jacobsen W, Kuhn B, Soldner A, Kirchner G, Sewing K-F, Kollman PA, et al. Lactonization is the critical first step in the disposition of the 3-hydroxy-3methylglutaryl-coa reductase inhibitor atorvastatin. Drug Metab Dispos. 2000; 28: 1369-1378.

13. Prueksaritanont $\mathrm{T}$, Subramanian $\mathrm{R}$, Fang $\mathrm{X}$, Ma $\mathrm{B}$, Qiu $\mathrm{Y}$, Lin $\mathrm{JH}$, et al. Glucuronidation of statins in animals and humans: A novel mechanism of statin lactonization. Drug Metab Dispos. 2002; 30: 505-512.

14. Pasanen MK, Fredrikson H, Neuvonen PJ, Niemi M. Different effects of SLCO1B1 polymorphism on the pharmacokinetics of atorvastatin and rosuvastatin. Clin Pharmacol Ther. 2007; 82: 726-733.

15. Keskitalo JE, Zolk O, Fromm MF, Kurkinen KJ, Neuvonen PJ, Niemi M. ABCG2 polymorphism markedly affects the pharmacokinetics of atorvastatin and rosuvastatin. Clin Pharmacol Ther. 2009; 86: 197-203.

16. Birmingham BK, Bujac SR, Elsby R, Azumaya CT, Wei C, Chen Y, et al. Impact of ABCG2 and SLCO1B1 polymorphisms on pharmacokinetics of rosuvastatin, atorvastatin and simvastatin acid in Caucasian and Asian subjects: A class effect? Eur J Clin Pharmacol. 2015; 71: 341-355.

17. Lee E, Ryan S, Birmingham B, Zalikowski J, March R, Ambrose $\mathrm{H}$, et al. Rosuvastatin pharmacokinetics and pharmacogenetics in white and Asian subjects residing in the same environment. Clin Pharmacol Ther. 2005; 78: 330-341.

18. Tsamandouras N, Dickinson G, Guo Y, Hall S, Rostami-Hodjegan A, Galetin A, et al. Identification of the effect of multiple polymorphisms on the pharmacokinetics of simvastatin and simvastatin acid using a population-modeling approach. Clin Pharmacol Ther. 2014; 96: 90-100. 
19. Tomita Y, Maeda K, Sugiyama Y. Ethnic variability in the plasma exposures of OATP1B1 substrates such as HMG-CoA reductase inhibitors: A kinetic consideration of its mechanism. Clin Pharmacol Ther. 2013; 94: 37-51.

20. Birmingham B, Bujac S, Elsby R, Azumaya C, Zalikowski J, Chen Y, et al. Rosuvastatin pharmacokinetics and pharmacogenetics in Caucasian and Asian subjects residing in the United States. Eur J Clin Pharmacol. 2015; 71: 329-340.

21. Peng KW, Bacon J, Zheng M, Guo Y, Wang MZ. Ethnic variability in the expression of hepatic drug transporters: Absolute quantification by an optimized targeted quantitative proteomic approach. Drug Metab Dispos. 2015; 43:1045-1055.

22. Keskitalo JE, Kurkinen KJ, Neuvonen PJ, Niemi M. ABCB1 haplotypes differentially affect the pharmacokinetics of the acid and lactone forms of simvastatin and atorvastatin. Clin Pharmacol Ther. 2008; 84: 457-461.

23. Wang D, Guo Y, Wrighton SA, Cooke GE, Sadee W. Intronic polymorphism in CYP3A4 affects hepatic expression and response to statin drugs. Pharmacogenomics J. $2011 ; 11: 274-286$.

24. Kivisto KT, Niemi M, Schaeffeler E, Pitkala K, Tilvis R, Fromm MF, et al. Lipidlowering response to statins is affected by CYP3A5 polymorphism. Pharmacogenetics. 2004; 14: 523-525.

25. Knauer MJ, Urquhart BL, Meyer zu Schwabedissen HE, Schwarz UI, Lemke CJ, Leake BF. Human skeletal muscle drug transporters determine local exposure and toxicity of statins. Circ Res. 2010; 106: 297-306.

26. Klein K, Thomas M, Winter S, Nussler AK, Niemi M, Schwab M, et al. PPARA: A novel genetic determinant of CYP3A4 in vitro and in vivo. Clin Pharmacol Ther. 2012; 91: 1044-1052. 
27. Knebel W, Gastonguay MR, Malhotra B, El-Tahtawy A, Jen F, Gandelman K. Population pharmacokinetics of atorvastatin and its active metabolites in children and adolescents with heterozygous familial hypercholesterolemia: Selective use of informative prior distributions from adults. J Clin Pharmacol. 2013; 53: 505-516.

28. Beal SL. Ways to fit a PK model with some data below the quantification limit. J Pharmacokinet Pharmacodyn. 2001; 28: 481-504.

29. Bergstrand M, Karlsson M. Handling data below the limit of quantification in mixed effect models. AAPS J. 2009; 11: 371-380.

30. Karlsson MO, Sheiner LB. The importance of modeling interoccasion variability in population pharmacokinetic analyses. J Pharmacokinet Pharmacodyn. 1993; 21: 735750.

31. Tessier A, Bertrand J, Chenel M, Comets E. Comparison of nonlinear mixed effects models and noncompartmental approaches in detecting pharmacogenetic covariates. AAPS J. 2015; 17: 597-608.

32. Lehr T, Schaefer H-G, Staab A. Integration of high-throughput genotyping data into pharmacometric analyses using nonlinear mixed effects modeling. Pharmacogenet Genomics. 2010; 20: 442-450.

33. Bertrand J, Balding DJ. Multiple single nucleotide polymorphism analysis using penalized regression in nonlinear mixed-effect pharmacokinetic models. Pharmacogenet Genomics. 2013; 23: 167-174.

34. Luna A, Nicodemus KK. Snp.Plotter: An r-based SNP/haplotype association and linkage disequilibrium plotting package. Bioinformatics. 2007; 23: 774-776.

35. Bertrand J, Comets E, Chenel M, Mentré F. Some alternatives to asymptotic tests for the analysis of pharmacogenetic data using nonlinear mixed effects models. Biometrics. 2012; 68: 146-155. 
36. Lindbom L, Pihlgren P, Jonsson N. PsN-Toolkit--a collection of computer intensive statistical methods for non-linear mixed effect modeling using NONMEM. Comput Methods Programs Biomed. 2005; 79: 241-257.

37. Narwal R, Akhlaghi F, Asberg A, Hermann M, Rosenbaum SE. Development of a population pharmacokinetic model for atorvastatin acid and its lactone metabolite. Clin Pharmacokinet. 2010; 49: 693-702.

38. Schwartz JB, Verotta D. Population analyses of atorvastatin clearance in patients living in the community and in nursing homes. Clin Pharmacol Ther. 2009; 86: 497502.

39. Poonkuzhali B, Lamba J, Strom S, Sparreboom A, Thummel K, Watkins P, et al. Association of breast cancer resistance protein/ABCG2 phenotypes and novel promoter and intron 1 single nucleotide polymorphisms. Drug Metab Dispos. 2008; 36: 780-795.

40. Cha P-C, Mushiroda T, Zembutsu H, Harada H, Shinoda N, Kawamoto S, et al. Single nucleotide polymorphism in ABCG2 is associated with irinotecan-induced severe myelosuppression. J Hum Genet. 2009; 54: 572-580.

41. Rudin CM, Liu W, Desai A, Karrison T, Jiang X, Janisch L, et al. Pharmacogenomic and pharmacokinetic determinants of erlotinib toxicity. J Clin Oncol. 2008; 26: 11191127.

42. Lemos C, Giovannetti E, Zucali PA, Assaraf YG, Scheffer GL, van der Straaten T, et al. Impact of ABCG2 polymorphisms on the clinical outcome and toxicity of gefitinib in non-small-cell lung cancer patients. Pharmacogenomics. 2011; 12: 159-70.

43. van Erp NP, Eechoute K, van der Veldt AA, Haanen JB, Reyners AKL, Mathijssen RHJ, et al. Pharmacogenetic pathway analysis for determination of sunitinib-induced toxicity. J Clin Oncol. 2009; 27: 4406-4412. 
44. Treviňo LR, Shimasaki N, Yang W, Panetta JC, Cheng C, Pei D, et al. Germline genetic variation in an organic anion transporter polypeptide associated with methotrexate pharmacokinetics and clinical effects. J Clin Oncol. 2009; 27: 59725978.

45. Ramsey LB, Panetta JC, Smith C, Yang W, Fan Y, Winick NJ, et al. Genome-wide study of methotrexate clearance replicates SLCO1B1. Blood. 2013; 121: 898-904.

46. Link E, Parish S, Armitage J, Bowman L, Heath S, Matsuda F, et al. SLCO1B1 variants and statin-induced myopathy-a genomewide study. N Engl J Med. 2008; 359: 789-799.

47. Sherry ST, Ward MH, Kholodov M, Baker J, Phan L, Smigielski EM, et al. dbSNP: The NCBI database of genetic variation. Nucleic Acids Res. 2001; 29: 308-311.

48. Bertrand J, Comets E, Mentre F. Comparison of model-based tests and selection strategies to detect genetic polymorphisms influencing pharmacokinetic parameters. J Biopharm Stat. 2008; 18: 1084-1102.

49. Bertrand J, Comets E, Laffont CM, Chenel M, Mentre F. Pharmacogenetics and population pharmacokinetics: Impact of the design on three tests using the SAEM algorithm. J Pharmacokinet Pharmacodyn. 2009; 36: 317-339.

50. Tsamandouras N, Dickinson G, Guo Y, Hall S, Rostami-Hodjegan A, Galetin A, et al. Development and application of a mechanistic pharmacokinetic model for simvastatin and its active metabolite simvastatin acid using an integrated population PBPK approach. Pharm Res. 2015; 32: 1864-1883.

51. Wendling T, Dumitras S, Ogungbenro K, Aarons L. Application of a Bayesian approach to physiological modelling of mavoglurant population pharmacokinetics. $\mathrm{J}$ Pharmacokinet Pharmacodyn. 2015; 42: 639-657. 
52. Tsamandouras N, Rostami-Hodjegan A, Aarons L. Combining the "bottom-up" and "top-down" approaches in pharmacokinetic modelling: Fitting PBPK models to observed clinical data. Br J Clin Pharmacol. 2015; 79: 48-55.

\section{Legends to Figures}

Fig. 1. ATA (left), o-OH-ATA (middle) and p-OH-ATA (right) plasma concentration data available for pharmacokinetic analysis. Study participants were on a regimen of $10 \mathrm{mg}$ atorvastatin once daily. The grey horizontal line represents the limit of quantification. The proportion of data below the limit of quantification (BQL) is also reported for each of the compounds.

Fig. 2. Structure of the joint ATA/o-OH-ATA pharmacokinetic model. $\mathrm{k}_{\mathrm{a}}$ : first-order absorption rate constant, $\mathrm{V}_{\mathrm{ATA}}$ : ATA volume of distribution, $\mathrm{V}_{\mathrm{o}-\mathrm{OH}-\mathrm{ATA}}$ : o-OH-ATA volume of distribution, $\mathrm{CL}_{\mathrm{ATA}}$ : ATA total elimination clearance, $\mathrm{CL}_{\mathrm{o}-\mathrm{OH}-\mathrm{ATA}}$ : o-OH-ATA total elimination clearance, $\mathrm{f}_{\mathrm{m}}$ : fraction metabolised to o-OH-ATA.

Fig. 3. Goodness of fit plots with regard to the ATA plasma concentration data. DV vs PRED and DV vs IPRED denote observations versus population and individual predictions respectively. CWRES vs TIME and CWRES vs PRED denote conditionally weighted residuals versus time and population predictions, respectively.

Fig. 4. Goodness of fit plots with regard to the o-OH-ATA plasma concentration data. DV vs PRED and DV vs IPRED denote observations versus population and individual predictions respectively. CWRES vs TIME and CWRES vs PRED denote conditionally weighted residuals versus time and population predictions, respectively. 
Fig. 5. Visual predictive check (VPC) of the developed population model for both ATA (left) and o-OH-ATA (right) plasma concentrations. In the upper panels, closed circles represent the observed plasma concentrations (study participants were on a regimen of $10 \mathrm{mg}$ atorvastatin once daily); highlighted with grey are the areas between the $5^{\text {th }}$ and $95^{\text {th }}$ percentiles of model simulations, whereas the red solid line represents the median; the horizontal dashed black line represents the limit of quantification. In the lower panels, red areas represent the simulation-based $95 \%$ confidence intervals for the fraction of modelsimulated samples below the limit of quantification (BQL) in each time bin, whereas closed circles represent the actual observed fraction of BQL samples in each time bin.

Fig. 6. Visual predictive check (VPC) of the developed population model stratified by the rs2622604 (ABCG2) genotype. Top and bottom panels refer to ATA and o-OH-ATA respectively. Left and right panels refer to the wild-type $(\mathrm{GG})$ and the variant genotype (AG) respectively. Closed circles represent the observed plasma concentrations (study participants were on a regimen of $10 \mathrm{mg}$ atorvastatin once daily); highlighted with grey are the areas between the $5^{\text {th }}$ and $95^{\text {th }}$ percentiles of model simulations, whereas the red solid line represents the median; the horizontal dashed black line represents the limit of quantification.

Fig. 7. Normalised histogram of the permutations likelihood ratio test statistics (change in objective function value) for the rs2622604 SNP overlaid with the probability density function of the $\chi^{2}$ distribution with 1 degree of freedom (in red). For the purpose of clarity the plot specifically focuses to the part of the histogram related to changes in objective function higher than 1.5 units. The insert plot further illustrates the Type-I error inflation as the permutation-corrected p-values (y-axis) are consistently higher than the p-values derived assuming that the test statistic follows a $\chi^{2}$ distribution with 1 degree of freedom (x-axis). 
Fig. 8. Linkage disequilibrium (LD) plot referring to the 7 SNPs tested in the SLCO1B1 gene in the studied Japanese population.

\section{List of Supplemental Digital Content}

Supplemental Digital Content 1. pdf 